\title{
The Conventional Prowess Termed Hemisection: 2 Case Reports
}

\author{
Seema Munjal ${ }^{1 *}$ and Sumit Munjal ${ }^{2}$
}

${ }^{1}$ Department of Prosthodontics and crown \& bridge, Dr. BR Ambedkar Institute of Dental sciences \& Hospital, Hariom nagar, Bihar, India ${ }^{2}$ Department of Periodontics, Career Post Graduate Institute of dental sciences \& Hospital, Lucknow, Uttar Pradesh, India

\begin{abstract}
Hemisection is a logical surgical option for treatment of a seemingly hopeless mandibular molar. Being a suitable alternative to extraction and implant therapy, it should be informed to each patient whenever indicated. The conservative demands have increased in dental practice and so have the pathological resorptions, root fractures and procedural accidents in endodontics. One case presented here was treated for furcation pathology and the other is reported with endodontic over instrumentation. A Perio- Prosthodontic management is demonstrated in both situations with an acceptable prognosis. For the reason that periodontal problems around resected molars have a tendency to recur, a sound diagnosis combined with a prudent case selection is a must.
\end{abstract}

Keywords: Hemisection, Resection, Furcation Involvement, Endodontic Mishap

\section{Introduction}

The dental clinician often experiences a mandibular molar that seems to be hopeless or the prognosis is guarded. Regenerative procedures such as Guided tissue regeneration are unpredictable in severe bony defects in relation to molars. ${ }^{[1]}$ In many situations, the resective option is justified to remove the affected part and save the tooth as a whole. This treatment modality i.e. hemisection, refers to sectioning of a mandibular molar into two halves followed by removal of the diseased root and its coronal portion. ${ }^{[2]}$ It is indicated where one of the root of molar is unsalvageable due to caries, Periodontitis or iatrogenic mishaps. ${ }^{[3]} \mathrm{A}$ complex canal anatomy, overzealous biomechanical preparation leading to ledging and perforations; and the use of NITI files are increasingly observed to cause an accidental separation. These instruments being flexible, unrealistic strains are placed upon them; especially in severely curved canals and at higher speeds. An increased risk of failure exists when a procedural accident occurs during treatment of infected teeth ${ }^{[4]}$ and thus can't be left unattended. The retained root is completed endodontically and restored later. In the presented cases referred to us, the patients were advised but deferred the decision to extract the tooth until an alternative option was suggested to them. Hemisectioning the retained deciduous molar is also practiced to manage the congenitally missing $2^{\text {nd }}$ premolar. ${ }^{[5]}$

\section{Case Report}

Case 1:A 40 years male patient reported to Faces $n$ braces research centre, New Delhi with food lodgement and intense pain on chewing in lower left back tooth since a fortnight. Neither the systemic involvement nor any extraoral abnormality was detected. He had a habit of frequent toothpicking on the affected site and traumatic occlusion was observed to be contributing to a $8 \mathrm{~mm}$ deep pocket around mesial root of 36 and the furca was involved both horizontally, as well as vertically when checked with Nabers probe and by transgingival sounding. (Figure 1a) Radiograph confirmed a 3 walled bony defect but the distal root showed a healthy bony support. (Figure $2 \mathrm{~b}$ ) No other anatomic defect could be judged on an IOPAR. Sectioning of the mesial root and crown was planned under local anaesthesia only after SRP, pulp extirpation, coronal obturation and detailing on the significance of oral hygiene maintenance. Following a cervical incision using no. 15 blade, the crown was carefully marked for resection with a diamond bur and lifted up along with the root.( Figure 1c,1d and 1e) Next the margins of remaining structure were smoothened with a finishing bur before the primary closure.(Figure 1f) The suture removal interval was used for endodontic completion. (Figure 1g) Finally, an extracoronal restoration was provided involving 35 and 36. Bone fill was evident on one year postoperative $\mathrm{X}$ ray and function of prosthesis was satisfactory to the patient. (Figure1h)

Case 2:A 45 year old female was referred to Faces $n$ braces research centre, New Delhi for the opinion on pain and swelling on right side of the face since 4 days. No correlation was found with the medical history but 47 was tender on percussion. RCT (Root canal treatment) was 
noticed to be attempted previously, which was confirmed on an intraoral radiograph (Figure2a), along with a NITI file separation in an apical perforation of the distal root. Another perforation was inspected to be in the furcation area. The procedural accident was patiently explained to her and two options offered; either to remove her tooth as a whole or to amputate the affected half. She decided for the later and a consent was obtained. The endodontic obturation was performed on the mesial root with simultaneous sulcular incision under appropriate anaesthesia, followed by splitting the distal portion (Figure $2 \mathrm{~b}$ and $2 \mathrm{c}$ ). Sutures were given after removing the same along with the broken instrument. (Figure2d) The postoperative X ray revealed favorable crown to root ratio(Figure2e) of the remaining structure, to which a post and core build up was done and finally a bridge given on 35, 36 and 37.(Figure2f)

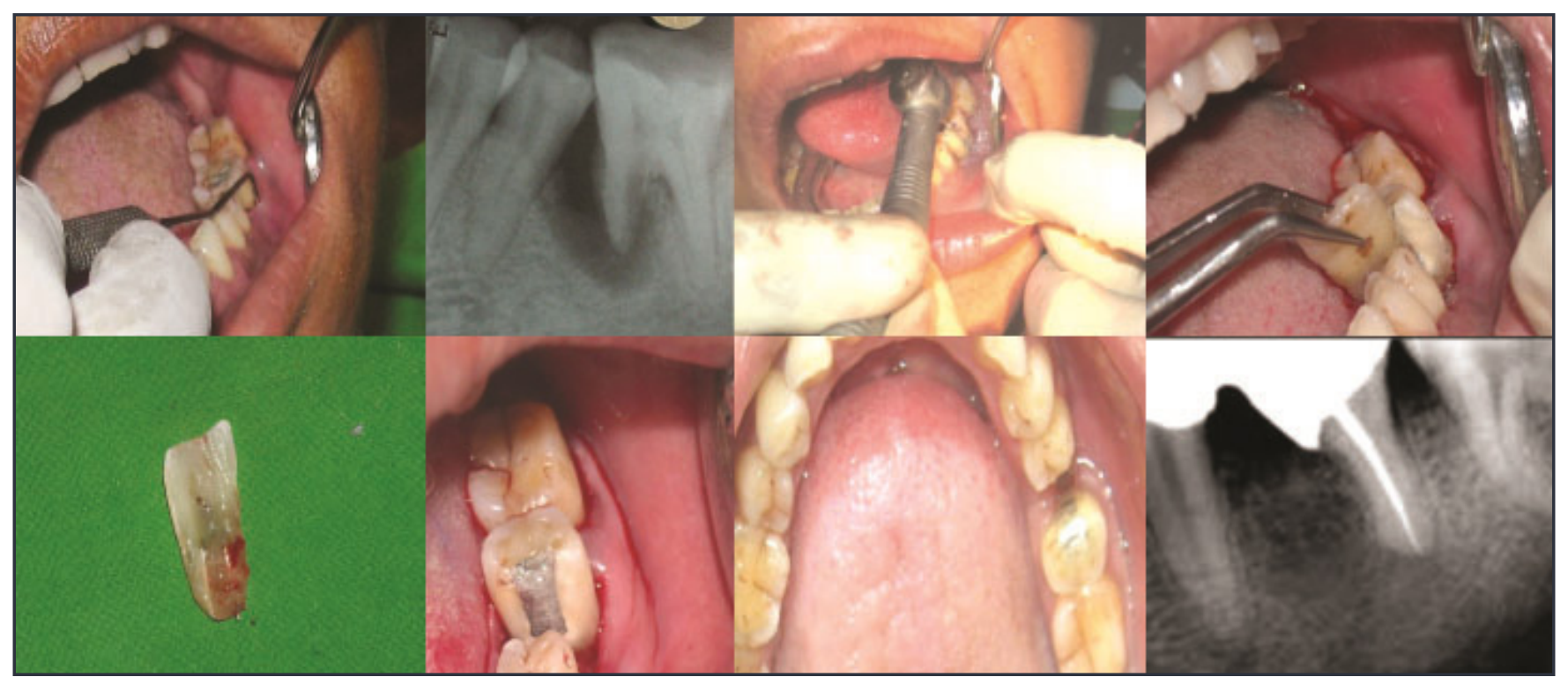

Fig. 1: case 1 (a) Preop view, (b.) Preop Xray, (c.) Splitting, (d.) Amputated portion, (e.) Resected root \& crown, (f.) Abutment smoothened, (g.) RCT completed, (h.) 1 year post op Xray.

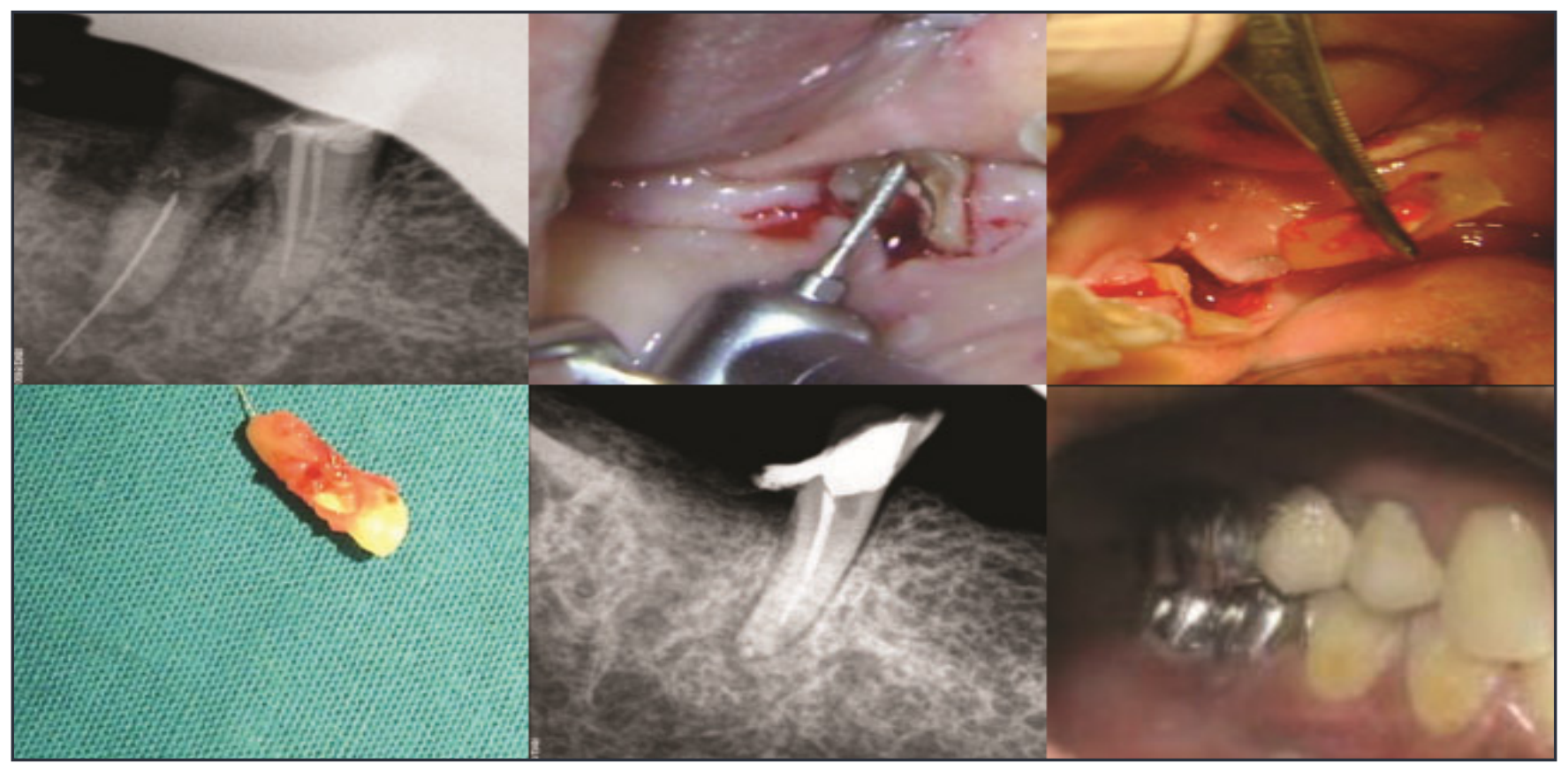

Fig. 2: case 2- (a.) Preop Xray, (b.) Incision and splitting, (c.) Amputated portion , (d.) Resected root \& crown, (e.) Postop Xray, (f.) Postop view. 


\section{Discussion}

The furcation per se is identified to be an area of complex morphology ${ }^{[6]}$ and the advances are welcome to treat root fractures, therapeutic misadventures and pathological resorption. Hemisection may be a suitable alternative to extraction and implant therapy and should be discussed with patients during consideration of treatment options.

${ }^{[3]}$ In most cases, the mesial root is removed owing to a longitudinal .groove where plaque control is inadequate and which decreases the surface area. A definite distal apical inclination and lesser curvature in distal root supports its retention in the technique. Mesial split was done in the one case as it was the diseased half and the distal was salvaged in the other since it was a victim of endodontic mishap. The conserved half serves as a load bearing abutment after final prosthesis. The long term survival rates in dental practice have been convincing in comparison to implants. ${ }^{[7,8]}$ However, there are a few contraindications as with all treatments. It is essential that root morphology allows for adequate surgical access, retained root is endodontically treatable and the final restoration is properly maintained. ${ }^{[3,9]}$ The greatest determinant of success of separated and prosthodontic restored teeth is baseline periodontal status ${ }^{[10]}$, which was assessed as satisfactory prior to the surgery in both the above cases. Interdental cleaning instructions should be reinforced at the completion for risk of caries in the resected area. Additionally, a follow-up may be planned to assess the occlusal forces and stress on abutment, which is prone to fracture, if the bridge is wide enough.

\section{Conclusion}

In the dilemma of implants Vs hemisection in dental disciplines, the later is an answer to the least risk-involved, most conservative and economically viable option to retain a compromised tooth. The overall prognosis has been evaluated and depends on many factors; but the quality of
RCT performed in surviving root, contours of restoration and effectiveness in plaque control are most decisive. The key to long term success lies with an eloquent diagnosist and going for a proper selection of the candidate.

\section{Reference}

1. Pontoriero R, Lindhe J. Guided tissue regeneration in the treatment of degree III furcations in maxillary molars. J Clin Periodontal 1995; 22; 810-812.

2. Kost WJ, Stakiw JE. Root amputation and hemisection. J Can Dent Assoc. 1991;57 (1):42-5.

3. Saad MN, Moreno J, Crawford C. Hemisection as an alternative treatment for decayed multirooted terminal abutment: a case report. J Can Dent Assoc. 2009; 75: 387-90.

4. Siqueira JF Jr. Aetiology of root canal treatment failure: why well treated teeth can fail. Int Endod J 2001;34 (1):1-10.

5. Northway WM. The nuts and bolts of hemisection treatment: managing congenitally missing mandibular second premolars. Am J Orthod Dentofacial Orthop. 2005; 127: 606-10.

6. Abdallah F, Kon S, Ruben MP. The furcation problem: etiology, diagnosis, therapy and prognosis. J West Soc periodontal 1987; 35: 129-41.

7. Buhler H. Survival rates of hemisected teeth: an attempt to compare them with survival rates of alloplastic implants. Int J Periodontics Restorative Dent. 1994;14: 536-43.

8. Fugazzotto PA. A comparison of the success of root resected molars and molar position implants in function in a private practice: results of up to 15-plus years. J Periodontol. 200; 72: 1113-1123.

9. Green EN. Hemisection and root amputation. J Am Dent Assoc 1986; 112: 511-18.

10. Zafiropoulos GG, Hoffmann O, Kasaj A, Willershausen B, Deli G, Tatakis DN. Mandibular molar root resection versus implant therapy: a retrospective nonrandomized study. J Oral Implantol. 2009;35: 52-62. 